\title{
A relevância do trabalho do enfermeiro na gestão em saúde, na prevenção de agravos e controle de custos em uma operadora de saúde
}

\author{
The importance of the nurse work in health management, disease prevention and cost \\ control in a health care provider
}

La relevancia del trabajo de las enfermeras en la gestión de la salud, la prevención de enfermedades y el control de costos en un proveedor de atención médica

Claudirene Milagres Araújo ${ }^{1 *}$, Felipe Fernandes de Oliveira ${ }^{1} J^{\prime}$ obelle Augusta Martins dos Santos ${ }^{1}$, Lorena Batista Silva ${ }^{1}$, Patrícia Eliza Miranda Dupim ${ }^{1}$, Brisa Emanuelle Silva Ferreira ${ }^{1}$, David Mattos de Andrade Ávila', Luciana Alves Silveira Monteiro².

\section{RESUMO}

Objetivo: Descrever a relevância do profissional enfermeiro em uma operadora de saúde, na gestão da prevenção de agravos e controle de custos através da telessaúde. Métodos: Trata-se de uma pesquisa exploratória, descritiva com abordagem qualitativa. O presente estudo foi realizado em uma operadora da rede suplementar de saúde, os dados foram coletados por meio de entrevistas, com 10 enfermeiros atuantes na telessaúde. Resultados: O estudo evidenciou o enfermeiro como profissional fundamental e responsável pela prevenção de agravos e redução de custos. Para os entrevistados o uso das tecnologias de informação aliadas ao conhecimento adquiridos sobre prevenção de agravos e promoção em saúde, gerou uma ferramenta onde o enfermeiro tem total autonomia para desenvolver atividades de sua competência e realizar o controle da carteira. Conclusão: Apesar das dificuldades por sua subvalorização, é possível perceber que o enfermeiro consegue se mostrar um gestor eficiente desenvolvendo ações modificadoras e permanentes que promovem saúde.

Palavras-chave: Telemedicina, Gestão em saúde, Promoção em saúde, Redução de custos, Educação em enfermagem.

\begin{abstract}
Objective: To describe the relevance of the professional nurse in a health care provider, in the management of disease prevention and cost control through telehealth. Methods: It is an exploratory, descriptive research with a qualitative approach. study was carried out in an operator of the supplementary health network, data were collected through interviews with 10 nurses working in telehealth. Results: This, study showed the nurse as a fundamental professional and responsible for the prevention of diseases and cost reduction. For the interviewees, the use of information technologies combined with the knowledge acquired about disease prevention and health promotion, generated a tool where nurses have full autonomy to develop activities within their competence and carry out portfolio control. Conclusion: Despite the difficulties due to its undervaluation, it is possible to see that nurses manage to show themselves as an efficient manager, developing permanent and modifying actions that promote health.
\end{abstract}

Keywords: Telemedicine, Health management, Health promotion, Cost reduction, Nursing education.

\footnotetext{
${ }^{1}$ Centro Universitário de Belo Horizonte (UniBH), Belo Horizonte - MG.

*E-mail: claudirene_milagres@hotmail.com

2 Faculdade Ciências Médicas, Belo Horizonte - MG.
} 


\section{RESUMEN}

Objetivo: Describir la relevancia del profesional de enfermería en un prestador de servicios de salud, en el manejo de la prevención de enfermedades y control de costos a través de tele salud. Métodos: Investigación exploratoria descriptiva con enfoque cualitativo. El estudio se llevó a cabo en un operador de la red complementaria de salud, los datos se recolectaron a través de entrevistas a 10 enfermeras que laboran en tele salud. Resultados: El estudio mostro al enfermero como un profesional fundamental y responsable de la prevención de enfermedades y reducción de costos. Para los entrevistados, el uso de tecnologías de la información combinado con los conocimientos adquiridos sobre prevención de enfermedades y promoción de la salud, generó uma herramienta donde los enfermeros tiene plena autonomia para desarrollar actividades dentro de su competencia y llevar a cabo el control de la cartera. Conclusión: A pesar de las dificultades por su infravaloración, es posible ver que los enfermeros logran mostrarse como un gestor eficiente, desarrollando acciones permanentes y modificadoras que promueven la salud.

Palabras clave: Telemedicina, Gestión de la salud, Promoción de la salud, Reducción de costos, Educación en enfermería.

\section{INTRODUÇÃO}

As atividades de saúde suplementar no Brasil iniciaram-se quando empresas do setor industrial começaram a ofertar assistência à saúde aos seus funcionários. O setor suplementar ficou sem regulamentação específica por vários anos, convivendo com o Sistema Único de Saúde (SUS) nascido em 1988 a partir da Constituição Federal. Apenas no ano de 1998 foi criada a Lei № 9.656 que regulamenta a oferta de planos de saúde privados. Posteriormente nasce a Agência Nacional de Saúde Suplementar (ANS), a partir da Lei ํㅜ 9.961, de 28 de janeiro de 2000, como instância reguladora das operadoras de assistência à saúde suplementar (MINISTÉRIO DA SAÚDE, 2021).

Assim, a qualidade do serviço prestado ao público e o custo gerado por procedimentos ofertados à saúde tem sido alvo de debate em diversas esferas. A economia em saúde e o processo de oferta do serviço de maneira satisfatória e de qualidade estão sendo colocadas em discussão numa tentativa de conciliação de ambos os processos. Contudo, vale lembrar que a oferta do serviço de saúde a determinada população é definida através de todos os aspectos, singularidades e demandas atribuídas ao perfil daquele público (CHAVES LDP e TANAKA OYO, 2012).

Com o objetivo de mudança no modelo assistencial de saúde em vigor, buscando a melhoria na qualidade de vida dos beneficiários de planos de saúde, iniciou-se um movimento para desenvolvimento de programas de promoção da saúde e prevenção de riscos e doenças, visto que no cenário atual grande parte das patologias que acometem a população é passível de prevenção (FREIRE RMA, et al., 2016).

Dessa forma, o enfermeiro deve atuar de maneira dinâmica, mas ressaltando que esse processo apenas ocorrerá se houver a centralização do paciente no ambiente do cuidado, trazendo consigo a sua individualidade e trabalhando de maneira biopsicossocial, com a visão de que cada pessoa é única, dotada de uma necessidade diferente da outra. A ampliação da atividade profissional nessa área e o desenvolvimento do trabalho devem ser fomentados de forma singular, assumindo assim papel de relevância no controle de custos e na oferta de serviços de qualidade (LOPES R, et al., 2013).

Para que o gestor em saúde, em sua grande maioria enfermeiros, desenvolvam habilidades onde consigam transitar entre o meio político e o técnico, estes devem desenvolver habilidades ao longo da sua graduação e na especialização, possibilitando que possam agir de maneira eficaz no âmbito do trabalho (CHAVES LDP e TANAKA OYO, 2012).

Nesse contexto, o objetivo do trabalho foi descrever a relevância do profissional enfermeiro em uma operadora de saúde, na gestão da prevenção de agravos e controle de custos através da telessaúde. 


\section{MÉTODOS}

Uma pesquisa descritiva, de abordagem qualitativa, que busca explorar a relevância do profissional enfermeiro em uma operadora de saúde, na gestão da prevenção de agravos e controle de custos através da telessaúde. A abordagem qualitativa se preocupa, nas ciências sociais, com um nível de realidade que não pode ser quantificado ou mensurado através de dados ou tabelas numéricas (MINAYO MCS, 2014; MINAYO MCS, 2010).

Esta pesquisa foi desenvolvida em uma unidade da rede suplementar de plano de saúde da rede privada de Belo Horizonte. Os sujeitos são enfermeiros do setor de gestão em saúde, atuantes em uma unidade privada de uma operadora de plano de saúde na cidade de Belo Horizonte. Foram entrevistados enfermeiros do setor, sendo todos atuantes na gestão e no uso da telessaúde como uma das ferramentas tecnológicas de trabalho. Os enfermeiros realizam oacompanhamento da carteira de clientes através dos meios de informação da tecnologia e a estruturação em grupos de acordo com as patologias.

Os critérios de inclusão dos sujeitos na pesquisa foram: tempo mínimo de três meses de atuação no setor, para que exista uma percepção do profissional sobre o objetivo do estudo, e aceitação em participar do mesmo voluntariamente. Os critérios de exclusão utilizados foram: o tempo de atuação no setor inferior a três meses e desistência do entrevistado de participar do estudo durante e após a realização da entrevista.

Este projeto foi aprovado pelo Comitê de Ética do Centro Universitário de Belo Horizonte (Unibh) em Pesquisa pela Plataforma Brasil sob $0 \mathrm{n}^{\circ}$ do parecer 3.205.750, respeitando todas as recomendações da Resolução no 510/2016 do Conselho Nacional de Saúde sobre pesquisa envolvendo seres humanos.

Foi garantido o anonimato dos entrevistados, bem como esclarecida a proposta do estudo, através da leitura do Termo de Consentimento Livre Esclarecido (TCLE). Os sujeitos da pesquisa assinaram o termo juntamente com o entrevistador em duas vias, ficando uma via com o entrevistador e outra com cada sujeito participante.

A coleta dos depoimentos ocorreu nos meses de abril e maio de 2019, por meio de entrevista, a partir de um roteiro semiestruturado, contendo cinco perguntas que descrevem a relevância do enfermeiro na gestão em telessaúde na prevenção de agravos e controle de custos. As entrevistas foram realizadas individualmente, dentro do próprio setor de trabalho do entrevistado, com agendamento prévio de acordo com a disponibilidade do profissional, e tiveram em média 15 minutos de duração. Foi utilizado um gravador para coleta das informações, com autorização prévia dos depoentes. Os dados coletados foram transcritos na íntegra, sistematizados e posteriormente analisados. As falas foram nomeadas com o pseudônimo $E$ (Enfermeiro) e numeradas sequencialmente do 1 ao 10.

Logo após a fase de entrevistas, os depoimentos foram transcritos na íntegra, a fim de serem analisados para extração de seu conteúdo latente. Em seguida, os conteúdos aproximados foram agrupados em categorias, para a apresentação e discussão com a literatura.

A análise de Conteúdo de Bardin foi o referencial de análise dos resultados. Trata-se de um conjunto de técnicas que analisam as comunicações e que utiliza procedimentos sistemáticos e objetivos para descrever o conteúdo das informações, procurando conhecer aquilo que está por trás das palavras sobre as quais se debruça (BARDIN L, 2011).

Aos professores orientadores coube o papel de realizar a estruturação e fundamentação teórica da maneira de realizar a pesquisa do aspecto qualitativo, além de realizar o cadastro do trabalho na plataforma Brasil, acompanhar o resultado do cadastro e as orientações e sugestões empregadas sobre o tema. Por fim, coube aos professores orientadores acompanhar a coleta de dados dos alunos participantes do processo de construção e elaboração da pesquisa.

Aos alunos ficou destinada a tarefa de realizar a coleta dos dados através das entrevistas dos participantes, bem como atender às solicitações feitas pelo CEP, organizar as entrevistas realizadas de maneira a estruturar e discutir de acordo com o embasamento científico apresentado ao longo de todo o processo. 


\section{RESULTADOS}

Os sujeitos entrevistados foram 10 enfermeiros do setor de gestão em saúde de uma operadora de saúde em Belo Horizonte, enfermeiros esses com experiências distintas quanto a visão em promoção a saúde e qualidade de vida ao beneficiário participante da carteira. As entrevistas foram divididas em quatro pontos principais, como demonstrado adiante:

\section{Melhoria na qualidade de vida dos usuários através da promoção em saúde}

Quando os entrevistados foram questionados sobre o papel do enfermeiro gestor em saúde na rede privada e a geração da melhoria da qualidade vida promovendo saúde relataram que os processos têm como finalidade levar ao beneficiário conhecimento sobre a sua saúde e a melhor forma de atingir o cuidado consciente.

"Eu demorei um pouco para perceber que isso não muda muito em relação rede pública, pois o foco é o mesmo a promoção à saúde. Atuar junto beneficiário, com o objetivo de melhorar a qualidade de vida e trazer benefícios à saúde [..]" (E2).

\section{Uso da tecnologia de informação na saúde}

Ao discorrer sobre o processo realizado dentro do setor, muitos profissionais deram ênfase à maneira como beneficiários de locais distintos do Brasil podem ser acompanhados e direcionados para a melhor estratégia do cuidado prestado, com o intuito de gerenciamento da carteira.

"Focamos em uma avaliação para direcionar os pacientes para o tratamento certo [...] ter acesso a um atendimento mais rápido e apropriado, para diminuir o número de internações e complicações." (E1).

O trabalho de promoção em saúde está sendo colocado em ênfase e discutido nas diversas esferas de saúde da rede privada como fio norteador do processo de cuidar do beneficiário, visando gerenciar o mesmo de forma holística e integral. Diante do exposto, pode-se perceber que o enfermeiro da rede privada, em relação ao trabalho de gestão em saúde, está cada vez mais inserido dentro da perspectiva do desenvolvimento da qualidade de vida e melhoria dos processos saúde/doença.

\section{A visibilidade do enfermeiro no âmbito profissional}

Ao longo da coleta de dados observou-se a comparação entre a autonomia do enfermeiro nos programas de promoção à saúde da Rede Pública e o da Rede Privada. Foram vários os relatos expostos pelos profissionais de saúde entrevistados nos quais são feitas comparações entre as ferramentas de gestão utilizadas na rede privada que auxiliam no trabalho do enfermeiro e melhoram o resultado com os beneficiários do plano de saúde, promovendo uma melhor percepção deste usuário sobre o papel do enfermeiro no processo do cuidado, em relação ao que ocorre na rede pública.

"Minha percepção é conseguimos ter um trabalho mais amplo com mais recursos $[\ldots]$ a gente tem um pouco mais de recurso embora seja preciso reduzir custo [...]" (E4).

"Dentro da rede privada, talvez, tenhamos uma estrutura melhor de trabalho [...] Na rede privada as coisas são mais ajustadas" (E5).

Entretanto percebeu-se que, mesmo com toda estrutura que a rede privada possa oferecer, grandes obstáculos foram citados pelos entrevistados quanto à percepção dos usuários sobre a real importância de um enfermeiro e sua atuação.

"Uma barreira a ser considerada é que o enfermeiro na rede privada não detém de maior autonomia como acontece na rede pública, onde ele pode ser gerente e coordenar um centro de saúde por exemplo. Na rede privada é preciso construir isso aos poucos, pois o paciente não entende que ele pode ter uma consulta de 
enfermagem, o enfermeiro é considerado como uma simples pessoa para fazer injeção, ou um curativo e isso se torna uma barreira de atendimento, porque a consulta de enfermagem é muito mais do que ser só um enfermeiro assistencial [...] “ (E3).

Foi notória a ênfase dada à forma como o enfermeiro é visto na rede privada. Muitos beneficiários, segundo relatos dos entrevistados, não sabem o real papel do enfermeiro como parte da equipe de saúde, acreditando tratarem-se apenas de um complemento de auxílio inferior.

Entretanto, segundo os entrevistados, existe a necessidade de um maior investimento e credibilidade por parte da rede privada como um todo em relação ao trabalho exercido pelo enfermeiro no âmbito da promoção em saúde. Pode-se perceber que quando o trabalho do enfermeiro é voltado para gestão da rede privada e para processos de promoção à saúde e controle de agravos, em uma operadora de saúde, não lhe é dada toda a autonomia necessária para execução de suas atribuições. O profissional de enfermagem pode desenvolver ações através dos seus conhecimentos e práticas que facilitariam o melhor direcionamento do beneficiário aos programas e ao cuidado em saúde.

\section{Uso da tecnologia da informação na saúde}

Os participantes ressaltaram a relevância do uso das tecnologias como forma de facilitar a integração dos usuários ao sistema de saúde, levando para eles o cuidado de forma mais ágil e profícua. Esta relação, gerada pelo uso das tecnologias, foi assim revelada:

"O uso das tecnologias é uma forma para acompanhar mais de perto o beneficiário através do telemonitoramento, pois é possível fazer uma busca ativa” (E6).

Os entrevistados reforçaram que essa modernização ao mesmo tempo em que se mostra muito positiva, em contrapartida se apresenta como uma alternativa de alto custo, no que diz respeito a investimento geral, e que não se tem um investimento específico para promoção à saúde. E claro na fala dos entrevistados que ainda se tem muito a construir para que estas tecnologias sejam mais voltadas para cuidado aos usuários.

A introdução de mecanismos de tecnologia como a telessaúde, que auxilia na interação do usuário com a equipe de saúde, é colocada como alternativa econômica em alguns aspectos pelos entrevistados.

O uso das tecnologias é um recurso que pode trazer dificuldades, mas que se mostra positivo na melhoria do cuidado ao beneficiário do plano.

\section{Redução de custos consecutiva ao trabalho de promoção à saúde}

O alcance de lucros é fator necessário diante de uma economia capitalista para assegurar o êxito de qualquer empreendimento. Isso não é diferente nas operadoras de saúde suplementar, que buscam ofertar serviços de qualidade mesmo que estes possam produzir alto custo. No entanto, estes serviços concomitantemente devem ser capazes de gerar provento.

"Acolhimento do paciente e da família promovendo um atendimento com orientações diferenciadas e com qualidade" (E10).

Por isso é necessário a atuação de um profissional habilitado que seja capaz de planejar e gerenciar a metodologia de promoção em saúde, com o intuito de melhoria da qualidade de vida dos seus usuários, centralizando o paciente no cuidado e, de maneira biopsicossocial, proporcionando diminuição de reinternações e na desestabilização das patologias crônicas, conforme apontado pelos entrevistados.

"[...] prevenção de riscos, agravos de doenças e aumento da qualidade de vida dos usuários [...] Um auxílio para operadora quanto a diminuição de custos” (E1).

A rede de saúde suplementar está despertando para a conveniência de que, para o indivíduo, prevenir é melhor do que reabilitar e/ou tratar, além de ser financeiramente vantajoso para a própria operadora de saúde. Investindo em promoção a saúde e alcançando estabilização do quadro dos pacientes, através de um acompanhamento próximo ao beneficiário, consegue-se não só alcançar melhorias. 


\section{DISCUSSÃO}

Observou-se que o uso de ações intersetoriais e educação em saúde para incrementar a consciência sanitária, pode modificar as condições de vida englobando o conceito de promoção à saúde (HEIDEMANN ITSB, et al., 2014).

Conforme este estudo, a rede de saúde suplementar estabelece modelagens estratégicas com ações programáticas integradas, com o intuito de prevenção de agravos, recuperação e reabilitação da saúde do beneficiário, atuando sob os princípios de integralidade e intersetorialidade.

Contudo, Freire RMA, et al. (2016), afirmam que com a de mudança no modelo assistencial de saúde, instaurou-se o desenvolvimento de programas de promoção à saúde e prevenção de riscos, visto que grande parte das patologiassão passíveis de prevenção.

Como se pode observar neste estudo, Ferreira GE, et al. (2017) ressaltam que a sistemática de promoção à saúde, é capaz de reduzir custos e atingir o desígnio maior de melhoria da qualidade de vida e saúde dos beneficiários, além da atuação de profissionais capacitados em elaborar e aplicar as metodologias de promoção à saúde e o controle de agravos das patologias que acometem a população abordada neste processo.

Destaca-se aqui a real participação do enfermeiro gestor como contribuinte e participante ativo para a melhoria da saúde dos beneficiários e a redução de custos através da diminuição das complicações e/ou internações hospitalares. Corroborando com este estudo, Silva MCN e Machado MH (2020) apontam que a formação com perfil multifuncional, característica da Enfermagem, possibilitou a esse professional uma maior possibilidade de cenários de atuação. Possibilitando a esse professional atuar tanto em procedimentos quanto na gestão.

Anselmi ML e Nakao JRS (2014) reafirmam que, historicamente ignorado desde o momento de sua institucionalização dentro de uma instituição de saúde, o profissional enfermeiro foi distanciado dos aspectos econômico-financeiros referentes à assistência de enfermagem, especialmente na rede privada, tornando-o subvalorizado e alienado das suas verdadeiras competências.

Neste trabalho ficou evidente a percepção dos entrevistados em relação à visibilidade do enfermeiro em seu cotidiano e ambiente de trabalho. De acordo com alguns deles, o trabalho do enfermeiro na rede privada ainda é alvo de desvalorização e sobrecarga de trabalho, tornando o profissional cansado e muitas vezes desmotivado. Martins MJD e Fernandes SJD (2014) dissertam que a visibilidade da enfermagem está ligada diretamente ao seu contexto histórico. Para que a enfermagem seja mais visível pela sociedade e pelos utentes do sistema de saúde, em abrangência e representatividade, cabe aos enfermeiros educar e difundir o conhecimento das suas ações e sua importância para o outro. Para que isso aconteça, segundo os autores, é necessária a internacionalização da produção científica da enfermagem de forma a promover o crescimento da enfermagem mundial.

Barbosa IAI e Silva MJD (2017) destacam em seu estudo que a telessaúde é uma ferramenta das tecnologias de informação que tem sido considerada uma importante ferramenta de trabalho para os enfermeiros devido ao seu importante papel desenvolvido através da escuta direta com o beneficiário. A interação gerada de ambos as partes e a diminuição da distância entre profissionais e pacientes têm culminado para um excelente resultado no âmbito de saúde. Concordando com esta afirmativa, a análise deste estudo aponta a telessaúde e/ou telemedicina como fator de maior comunicação com os usuários, e relevante facilitador do trabalho realizado pelo enfermeiro, uma vez que, com o uso das tecnologias as informações ficam mais facilmente disponíveis e atualizadas.

Segundo Almino MFAB, et al. (2014) outro instrumento de rápido manejo e fácil transmissão de informações que vale ser salientado é o telemonitoramento, pois faz-se importante sua utilização pela dificuldade na aproximação do serviço de saúde dos usuários de diversas regiões do país.

Os dados deste estudo vão de encontro ao que afirmaram Almino MFAB, et al. (2014), expressando que os mecanismos de tecnologia como o telemonitoramento auxiliam na interação dos usuários com a equipe de saúde, e é uma alternativa que propicia uma economia em alguns aspectos. 
O enfermeiro dispõe de ferramentas e várias possibilidades de atuação no âmbito da gestão em saúde. Com o intuito de trabalhar com o beneficiário na promoção em saúde e controle de agravos, o profissional deve desenvolver ferramentas que, junto a isso, reduzam gastos com as possíveis intercorrências características do processo de gerência (CRUZ PL, et al., 2016).

O direcionamento adequado dos beneficiários foi considerado pelos participantes deste estudo como sendo uma forma positiva de realizar a melhor captação e controle da carteira do plano e, consequentemente, controlar as patologias crônicas dos usuários. Cruz PL, et al. (2016) sugerem que após a estruturação das metas e métodos que o enfermeiro irá aplicar na instituição, deverá haver uma análise e discussão destas metas e após as mesmas deverão ser apresentadas para todos os envolvidos no processo.

A forma como os beneficiários são encaminhados aos programas de acompanhamento e controle das patologias que possuem em comum, o tipo de abordagem e direcionamento, além dos aspectos epidemiológicos tratados, facilitam o processo de captação desses usuários, e com isso, proporcionam uma maneira mais eficaz de promover o controle dos agravos. Para Cruz PL, et al. (2016) o enfermeiro deve atuar como transformador da realidade do processo, desenvolver os projetos, estabelecer indicadores e alcançar a melhoria da qualidade do atendimento.

Corroborando com este estudo em que os entrevistados enaltecem sempre o aprimoramento dos aspectos individuais de cada usuário, mesmo fazendo parte de grupos com a mesma característica clínica. Segundo Pereira LA, et al. (2015) em qualquer nível institucional, a gestão na saúde, encontra vários desafios que precisam ser enfrentados. O enfermeiro gestor enfrenta várias situações e problemas, de naturezas diversas, que podem ser abordados de várias maneiras, dependendo de combinações entre técnicas/métodos e tecnologias/equipamentos disponíveis para a organização dos processos de trabalho.

\section{CONCLUSÃO}

Este estudo demonstra a relevância do trabalho do enfermeiro gestor em telessaúde e o uso das ferramentas tecnológicas como meio de propagação de informações voltadas para promoção de saúde e controle de agravos, gerando um maior controle da carteira de beneficiários e o controle de custos. Foi perceptível a importância do enfermeiro na operadora de saúde, junto ao processo de trabalho desenvolvido em promoção à saúde e o controle de agravos visando a melhor a qualidade de vida. $\mathrm{O}$ uso das tecnologias de informação foi destacado como peça chave de interlocução com o usuário e a demanda de saúde apresentada. Além disso, há a percepção da execução do trabalho preventivo e de rastreio, antes realizado apenas na rede primária do SUS, sendo implantada de maneira efetiva por enfermeiros na rede suplementar de saúde, abordando o beneficiário antes que ocorra o adoecimento e/ou o agravo.

\section{REFERÊNCIAS}

1. ALMINO MAFB, et al. Telemedicina: um instrumento de educação e promoção da saúde pediátrica. Revista Brasileira de Educação Médica. Fortaleza -CE, 2014; 38(3): 397-402.

2. ANSELMI ML, NAKAO JRS. A enfermagem no processo de gestão econômica dos serviços de saúde: limites e possibilidades. Revista Brasileira de Enfermagem. Brasília, 2014; 5(2): 223-232

3. BARBOSA IAI, PAES MJDS. Cuidado de enfermagem por telessaúde: qual a influência da distância na comunicação? Revista Brasileira de Enfermagem, 2017; 70(5): 978-984.

4. BARDIN L. Análise de Conteúdo. 6 ed. São Paulo: Almedina, 2011, 288p.

5. CHAVES LDP, TANAKA OY. O enfermeiro e a avaliação na gestão de Sistemas de Saúde. Rev. esc. enferm. USP [online]., 2012; 46(5): 1274-1278.

6. COSTA VT, et al. Melhores práticas do enfermeiro gestor no gerenciamento de risco. Revista Latino-Americana de Enfermagem, 2013; 21(5): 1165-1171.

7. CRUZ PL, et al. Os Desafios do Enfermeiro Gestor nos Serviços em Saúde. Revista Gestão em Foco, 2016 : 1- 7.

8. FERREIRA GE, et al. Perspectivas de enfermeiros da rede hospitalar privada acerca da gestão de pessoas: análise da psicodinâmica do trabalho. Revista de Administração em Saúde, 2017; 17(69).

9. FREIRE RMA, et al. Um olhar sobre a promoção da saúde e a prevenção de complicações: diferenças de contextos. Revista Latino-Americana de Enfermagem. São Paulo, 2016; 24: 1-9. 
10. HEIDEMANN ITSB, et al. Promoção da Saúde na Atenção Básica: estudo baseado no método de Paulo Freire. Ciência \& Saúde Coletiva, 2014; 19: 3553-3559.

11. LOPES R, et al. Telemonitoramento de pacientes hipertensos e diabéticos: Revisão integrativa. In: Seminário Nacional de Pesquisa de Enfermagem. Pernambuco, 2013: 1929-1930.

12. MARTINS MJR, FERNANDES SJD. A visibilidade da enfermagem, dando voz à profissão: revisão integrativa. Revista de Enfermagem UFPE online, 2014; 8(1): 2422-2433.

13. MINAYO MC. Apresentação. In R. Gomes, Pesquisa qualitativa em saúde. São Paulo: Instituto Sírio Libanes, 2014.

14. MINAYO MC. O desafio do conhecimento. São Paulo: Hucitec, 2010; 416p.

15. MINISTÉRIO DA SAÚDE. Agência Nacional de Saúde Suplementar. Quem somos: histórico. Brasil, 2021. Disponível em: https://www.gov.br/ans/pt-br/acesso-a-informacao/institucional/quem-somos-1/historico.

16. MINISTÉRIO DA SAÚDE. Agência Nacional de Saúde Suplementar. Resolução Normativa no 264/11. Dispõe sobre a promoção da saúde e prevenção de riscos e doenças e seus programas de saúde suplementar. Brasil, 2011. Disponível em: https://bvsms.saude.gov.br/bvs/saudelegis/ans/2011/res0264_19_08_2011.html. Acesso em: 19 dez. 2021.

17. MINISTÉRIO DA SAÚDE. Portal de Saúde - SUS. Brasil, 2021. Disponível em: http://portalsaude.saude.gov.br. Acesso em: 08 dez. 2021.

18. PEREIRA LA, et al. Enfermagem e liderança: percepções de enfermeiros gestores de um hospital do sul do Brasil. Rev pesqui cuid fundam online. 2015: 7(1):1875-1882.

19. PIROPO TGN, AMARAL HOS. Telehealth, contexts and implications in Bahia scenario. Saúde em Debate, 2015; 39(104): 279-287.

20. SILVA MCN, MACHADO MH. Sistema de Saúde e Trabalho: desafios para a Enfermagem no Brasil. Revista Ciência saúde coletiva. Rio de Janeiro, 2020; 25(1). 